\title{
MATHEMATICAL ANALYSIS USING TWO MODELING TECHNIQUES FOR DYNAMIC RESPONSES OF A STRUCTURE SUBJECTED TO A GROUND ACCELERATION TIME HISTORY
}

\author{
YONG-WOO KIM ${ }^{1 *}$ and MYUNG JO JHUNG ${ }^{2}$ \\ ${ }^{1}$ Department of Mechanical Engineering, College of Engineering, Sunchon National University \\ Suncheon, Jeonnam, Korea 540-742 \\ ${ }^{2}$ Safety Research Division, Korea Institute of Nuclear Safety \\ 19 Guseong-dong, Yuseong-gu, Daejeon, Korea 305-338 \\ ${ }^{*}$ Corresponding author. E-mail : kyw@sunchon.ac.kr \\ Received October 29, 2010 \\ Accepted for Publication March 29, 2011
}

Two types of numerical modeling techniques were considered for the dynamic response of a structure subjected to a ground acceleration. One technique is based on the equation of motion relative to ground motion, and the other is based on the equation of absolute motion of the structure and the ground. The analytic background of the former is well established while the latter has not yet been extensively verified. The latter is called a large mass method, which allocates an appropriate large mass to the ground so that it causes the ground to move according to a given acceleration time history. In this paper, through the use of a single degree-of-freedom spring-mass system, the equations of motion of the two techniques were analyzed and useful theorems are provided on the large mass method. Using simple examples, the numerical results of the two modeling techniques were compared with analytic solutions. It is shown that the theorems provide a clear insight on the large mass method.

KEYWORDS : Large Mass Method, Additional Force Model, Large Mass Model, Ground Acceleration Time History, Dynamic Response, Seismic Load

\section{INTRODUCTION}

The structures and equipment in a nuclear power plant are required to resist seismic loads, which are transmitted directly from the ground to the entire structure in the form of vibratory loads. According to Reference [1], there are three analytical methods and two numerical methods that are used in the dynamic analyses of structures subjected to ground acceleration. The three analytic methods are the quasi-static decomposition method ${ }^{[2]}$, the integral representation with the Cesaro sum technique ${ }^{[1]}$, and the integral representation with the Stokes' transformation ${ }^{[3]}$. The two numerical methods are the large mass FEM simulation technique and the large stiffness FEM simulation technique. The numerical techniques are easily incorporated into commercial programs to solve the problem. Chen et al. ${ }^{[1]}$ reported that the results obtained using the five methods are in good agreement for a Rayleigh-damped Bernoulli-Euler beam. However, the authors in Reference [1] considered a structure subjected to given ground displacements while this paper considers a structure subjected to given ground acceleration.

When the ground motion is recorded in the form of an acceleration time history, the most preferred and convenient way for direct dynamic analyses is the large mass method because it enables the acceleration time history to be input easily. Thus, if the dynamic response analyses are performed using the measured ground acceleration time history, the acceleration time history must be transformed to the corresponding loading time history for the direct dynamic analyses. To accomplish this, many commercial computer programs employ two types of methodologies: the large mass method and the method using relative motion with respect to the ground. ${ }^{[4-6]}$ The first method is preferred by many users because it is considered that the FEM requires forces or displacements as input data, which is true and the use of acceleration as input data is rare. However, many users still debate the validity of the large mass method because its analytical background has not yet been sufficiently described. The following questions typically asked by users: 
- Is it a standard method to use the acceleration time history when applying a force to the large mass?

- How do you calculate the mass participation ratio or how sensitive is the magnitude of the large mass that is used?

- When does a problem with a round-off error begin? In this paper, the mathematical and numerical aspects of the two models are investigated in order to answer these questions, and numerical examples are used to illustrate and verify useful theorems in the large mass method.

\section{MATHEMATICAL ANAYSIS}

\subsection{Two Types of Equation of Motion}

Consider the simple model shown in Fig. 1, in which the ground is subjected to an acceleration time history $\ddot{z}(\mathrm{t})=a(t)$. The equation of motion is:

$$
m \ddot{v}+c(\dot{v}-\dot{z})+k(v-z)=0,
$$

where $m, c$, and $k$ denote the structural mass, damping coefficient, and spring constant, respectively. In addition, $v$ and $z$ are the displacements of the structure and the ground, respectively.

Equation (1) can be rewritten as:

$$
\begin{aligned}
& \quad m(\ddot{v}-\ddot{z})+c(\dot{v}-\dot{z})+k(v-z)=-m \ddot{z}, \\
& \text { or } \quad m \ddot{w}+c \dot{w}+k w=-m \ddot{z}, \\
& \text { or } \quad m \ddot{w}+c \dot{w}+k w=f(t),
\end{aligned}
$$

where $w=v-z$, which is the relative displacement of the structure with reference to the ground, and $f(t)=-m a(t)$. Equation (3) can be interpreted as the equation of motion of the system shown in Fig. 2, where the ground is fixed and the structural mass, $m$, is subjected to the force, $f(t)=$ $-m a(t)$. For this reason, the model presented in Fig. 2 is referred to as the 'additional force' model (or AF model) in this study. Using this model, the dynamic responses of the relative motion of the structure can be obtained with reference to the ground.

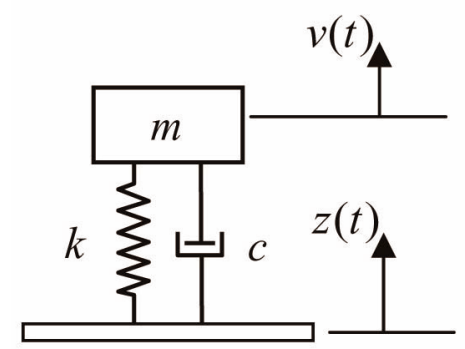

ground

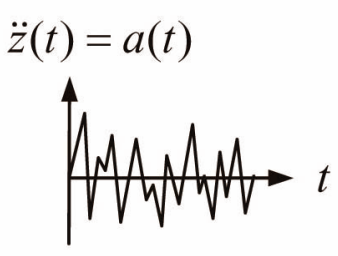

Fig. 1. Original Model under Consideration
The 'large mass' model (or LM model) is also used to solve the same problem. To make the ground move in accordance with the given acceleration $a(t)$, the LM model introduces a ground mass as shown in Fig. 3, where $M$ denotes the ground mass. However, it is not expected that the ground mass moves with the given acceleration $a(t)$ due to the spring and damper above the ground mass. Nevertheless, the ground can follow the acceleration approximately if an appropriately large magnitude of ground mass is chosen. According to many researchers and engineers, there is a specific magnitude of the ground mass $(M)$ that may yield a reasonable dynamic response. Then, some questions arise including how large the mass should be to be appropriate for the ground mass and why this magnitude of the ground mass exists.

Consider the large mass model in Fig. 3. The equation of motion is:

$$
\left[\begin{array}{cc}
m & 0 \\
0 & M
\end{array}\right]\left\{\begin{array}{l}
\ddot{v} \\
\ddot{z}
\end{array}\right\}+\left[\begin{array}{cc}
c & -c \\
-c & c
\end{array}\right]\left\{\begin{array}{l}
\dot{v} \\
\dot{z}
\end{array}\right\}+\left[\begin{array}{cc}
k & -k \\
-k & k
\end{array}\right]\left\{\begin{array}{c}
v \\
z
\end{array}\right\}=\left\{\begin{array}{c}
0 \\
F(t)
\end{array}\right\},
$$

where $F(t)=M a(t)$.

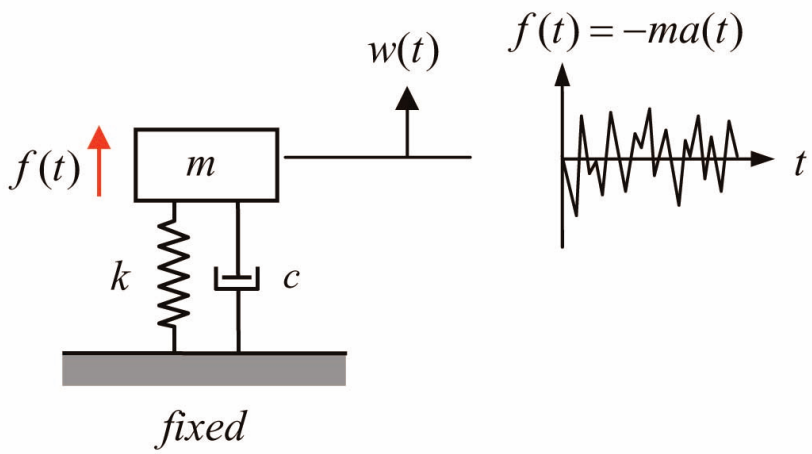

Fig. 2. The AF Model that Corresponds to the Model in Fig. 1

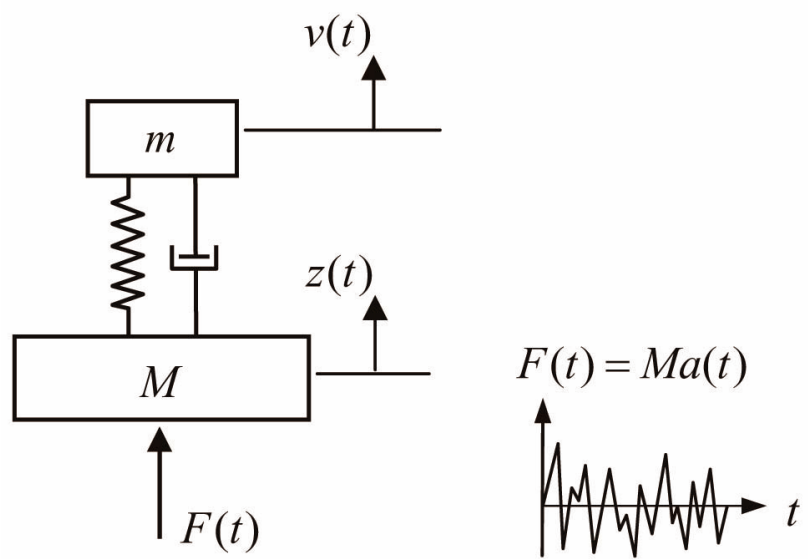

Fig. 3. The LM Model that Corresponds to the Model in Fig. 1 


\subsection{Mathematical Analysis on Behavior of the LM Model}

To consider the effect of the inertia terms only, by summing the two equations in equation (4) the following equation is obtained:

$$
M \ddot{z}+m \ddot{v}=M a .
$$

Dividing both sides of equation (5) by $M a(\neq 0)$, the following is obtained:

$$
\frac{\ddot{z}}{a}+\frac{\ddot{v}}{(M / m) a}=1,
$$

where the mass ratio of $M / m$ is called the 'large mass ratio' in this paper. A graph of equation (6) is plotted in Fig. 4.

The accelerations of the structural mass $(m)$ and the ground mass $(M)$ should be in line with equation (6) in Fig. 4. It can be seen that $M / m \rightarrow \infty$ to be $\ddot{z} \rightarrow a$ from equation (6).

Assume that $a(\neq 0)$ is a finite value. Then, $\ddot{z}$ and $\ddot{v}$ have finite values. Under this assumption, the following theorems can be drawn.

[Theorem 1]

As $M / m$ increases, $\ddot{z}$ approaches $a$.

[Theorem 2]

If $M / m$ is so large that $\ddot{z} \rightarrow a$, then $\frac{\ddot{v}}{(M / m) a} \rightarrow 0$ but $\ddot{v} \neq 0$.

[Theorem 3]

If $M / m$ is so large as to make $\ddot{v} \equiv 0$, then $\ddot{z} \equiv a$.

[Theorem 4]

If $M / m$ is small, then $\ddot{z} \neq a$ unless $\ddot{v}=0$.
Theorem 1 says that the large mass ratio of $M / m$ should be large for $\ddot{z}$ to approach the given ground acceleration (a). However, it does not state the size of the mass ratio.

Theorem 2 and Theorem 3 state that an appropriate large mass ratio, $M / m$, exists that induce $\ddot{z} \rightarrow a$ and $\ddot{v} \neq 0$. However, if $\ddot{v}=0, \ddot{z}$ will be the same as the ground acceleration from equation (6), which is described by Theorem 3. This situation can occur in a numerical calculation as a result of numerical errors. That is, a value of $M / m$ that is too large could produce a zero acceleration of the structure with the assistance of a numerical error. Numerical errors occur due to the finite number of bits. Thus, the upper limit of $M / m$ depends on the machines or programs used.

Theorem 4 states that the acceleration of the ground mass cannot be reproduced if the mass ratio $\mathrm{M} / \mathrm{m}$ is small. Thus, when understood together, Theorem 3 and Theorem 4 state that if the values of the large mass ratio are too small or too large, it can cause significant errors in the calculation of the accelerations, $\ddot{z}$ and $\ddot{v}$.

Now, consider the behavior of the accelerations under the situation described in Theorem 2. The first part of equation (4) is given as:

$$
\begin{aligned}
& \quad m \ddot{v}+c(\dot{v}-\dot{z})+k(v-z)=0, \\
& \text { or } m(\ddot{v}-\ddot{z})+c(\dot{v}-\dot{z})+k(v-z)=-m \ddot{z}, \\
& \text { or } m \ddot{w}+c \dot{w}+k w=-m \ddot{z} .
\end{aligned}
$$

The second part of equation (4) is:

$$
M \ddot{z}+c(\dot{z}-\dot{v})+k(z-v)=M a .
$$

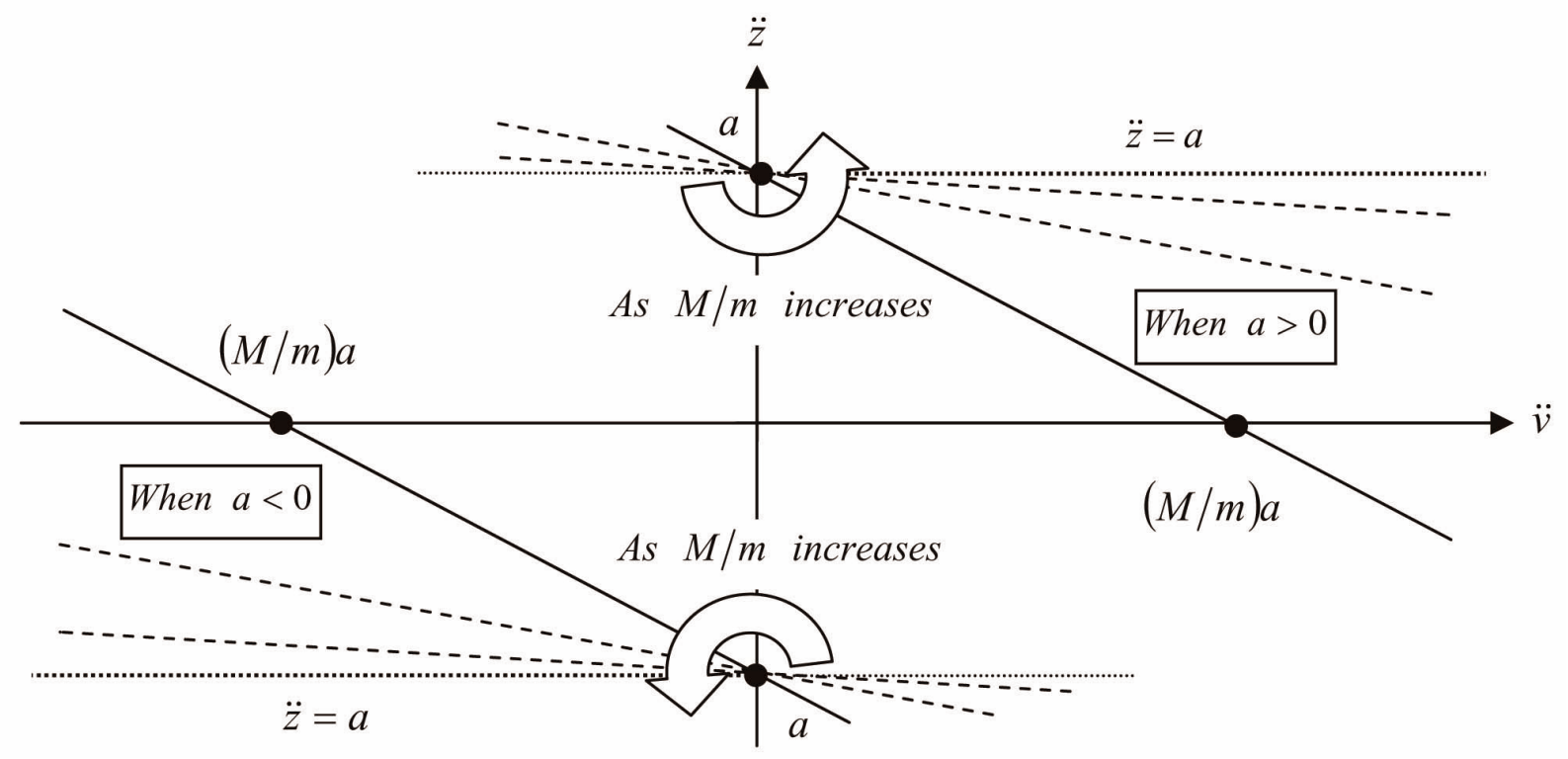

Fig. 4. Graph of Equation (6) 
Substituting equation (8) into equation (10), the following is obtained:

$$
M \ddot{z}+m \ddot{v}=M a,
$$

which is the same as equation (5) that was considered as the starting point for the analysis.

When the large mass ratio is sufficiently large as described in Theorem 2, that is, under the conditions of $\ddot{z} \rightarrow a$ and $\ddot{v} \neq 0$, equation (9) can be written as:

$$
m \ddot{w}+c \dot{w}+k w \approx-m a(t),
$$

which is the approximate equation of motion of the $\mathrm{AF}$ model. The above analysis demonstrates how the LM model can yield good approximate solutions when the large mass ratio is of a sufficient magnitude to make $\ddot{z} \rightarrow a$ and $\ddot{v} \neq 0$. Thus, the following theorem can be asserted.

\section{[Theorem 5]}

If $M / m$ is sufficiently large to make $\ddot{z} \rightarrow a$ and $\ddot{v} \neq 0$, the equation of motion of the LM model is reduced, approximately, to the equation of motion of the AF model. Therefore, an approximate solution can be obtained under the conditions of $\ddot{z} \rightarrow a$ and $\ddot{v} \neq 0$. Note that the solutions obtained using the LM model are not exact solutions but rather approximate ones.

\subsection{Consideration of the Effect of the Stiffness and Damping Coefficient}

According to the analysis in the previous section, the accuracy of the LM model's solutions depends on the large mass ratio because the solutions of the LM model should satisfy equation (5). In this section, the influence of the magnitudes of $k$ and $c$ on the accuracy of the LM model's solution is investigated and it will be demonstrated that the accuracy of the LM model's solution only depends on the large mass ratio.

Dividing both sides of the second equation in equation (4), the following is obtained:

$$
\ddot{z}+\frac{c}{M}(\dot{z}-\dot{v})+\frac{k}{M}(z-v)=a .
$$

For the ground mass in equation (12) to move in accordance with a given acceleration, $a(t)$, and for the velocity $(\dot{v}-\dot{z})$ and displacement $(v-z)$ in equations (7) or (8) not to be zero, the following conditions should be satisfied.

$$
\begin{aligned}
& c / M \rightarrow 0 \text { and } k / M \rightarrow 0, \\
& \text { or } \quad 2 \zeta \omega_{n}<<M / m \text { and } \omega_{n}{ }^{2}<<M / m,
\end{aligned}
$$

where $\zeta$ and $\omega_{n}=\sqrt{\mathrm{k} / \mathrm{m}}$ are the damping ratio and natural circular frequency of the corresponding AF model, respectively. Note that the conditions in equation (14) were obtained from the second equation of the equation of motion in the LM model. It appears that equation (13) or equation (14) should be satisfied in order for $\ddot{z} \rightarrow a$.
Now, consider the first equation of equation (4) in addition to the second equation:

$$
\begin{aligned}
& m \ddot{v}+c(\dot{v}-\dot{z})+k(v-z)=0, \\
& \text { or } m \ddot{v}=c(\dot{z}-\dot{v})+k(z-v) .
\end{aligned}
$$

Equation (16) implies that $m \ddot{v}$ represents the sum of the spring force and damping force. Thus, the second term $m \ddot{v}$ in equation (5) represents the sum of the spring force and damping force. Therefore, the effects of the spring force and damping force do not need to be considered once the large mass ratio is considered by equation (5). That is, the accuracy of the solutions obtained using the LM model depends on the large mass ratio irrespective of the conditions in equation (14), as stated in Theorem 6.

[Theorem 6]

The accuracy of the LM model's solution does not depend on the magnitudes of $c$ and $k$.

\section{NUMERICAL TESTS AND DISCUSSION}

In this section, the theorems are verified and the appropriate size range of the large mass ratio is determined using various numerical tests. This is undertaken by performing a dynamic response analysis using ANSYS, which employs the Newmark method ${ }^{[4,7]}$.

\subsection{Effect of the Large Mass Ratio in LM Model}

Figure 5 shows the original model and its equivalent AF and LM models. The analytical solutions are given in the Appendix.

At first, the effect of the mass ratio $M / m$ was tested in the LM model in Fig. 5(c) and the results are plotted in Table 1. From Table 1, it can be seen that $M / m=10^{\circ}$ yields erroneous responses because the mass ratio of $M / m$ is too small to reproduce the given ground acceleration $a(t)$, which was predicted using Theorem 1 and Theorem 4 . Physically, the responses for $M / m=10^{\circ}$ are interpreted as follows. The system is a 2-DOF semi-definite system that consists of two equal masses and one spring. Thus, one natural frequency is zero and the other is $\omega=\sqrt{k / 2 m}$. The eigenvectors are $\{z v\}=\{11\}$ and $\{1-1\}$. Thus, the system moves like a rigid body and the two masses are oscillating with the frequency $\omega=\sqrt{k / 2 m}$ when no external force exists (or after $t=1$ ).

The results of the LM model agree with the analytic solutions when the large mass ratio is in the range of $M / m=10^{3} \sim 10^{11}$. However, $M / m=10^{12}$ gives zero acceleration of the structure $(\ddot{v})$ even though the large mass ratio yields a good approximate motion of the ground mass $(\ddot{z}, \dot{z}$, and $z$ ). The results indicate that there is an appropriate range of the large mass ratio, which was predicted by Theorem 2 and Theorem 5 .

The numerical data of $\ddot{z}$ and $\ddot{v}$ during the time interval 


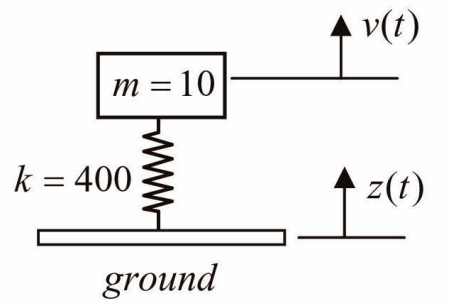

(a)

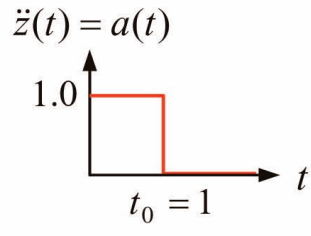

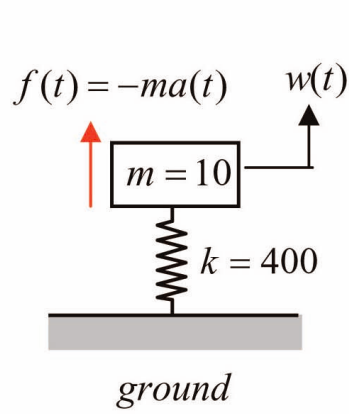

(b)

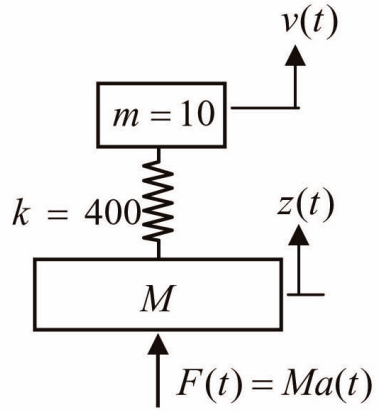

(c)

Fig. 5. (a) The Original Model Subjected to a Ground Acceleration Time History, (b) the AF Model, and (c) the LM Model

Table 1. Dynamic Responses of the LM Model in Fig. 5(c) Subjected to the Ground Acceleration in Fig. 5(a)

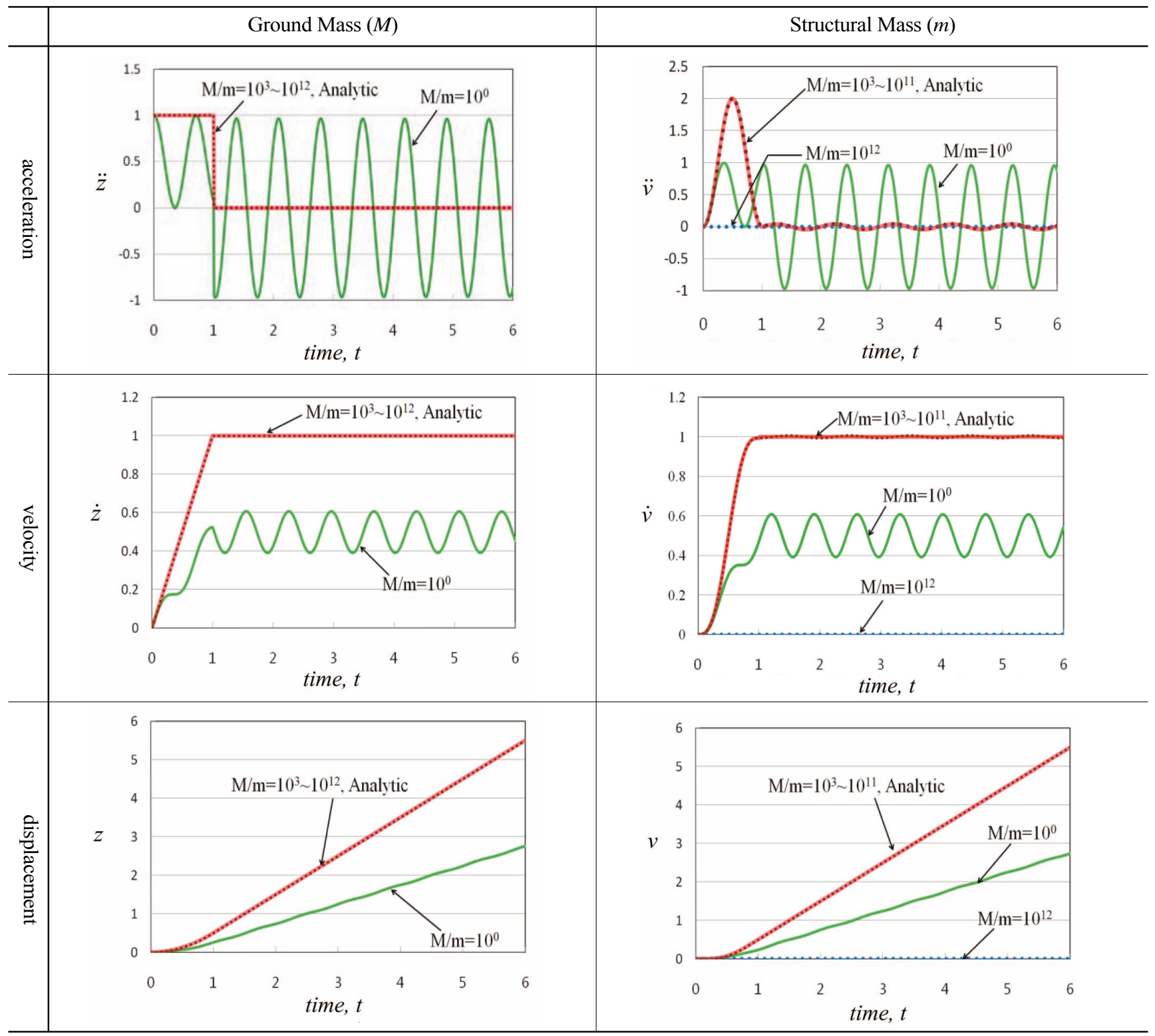


$0 \leq t \leq 1$, in which $a(t) \neq 0$, are tabulated in Table 2 for $M / m=10^{11}$ and $10^{12}$. The dramatic differences in the two data sets rationalize Theorem 3 .

The physical meaning of the LM model's solutions for $M / m \geq 10^{3}$ can be explained as follows. The LM model is a 2-DOF semi-definite system that consists of two unequal masses and one spring. Its natural frequencies are $\omega_{1}=0$ and $\omega_{2}=\sqrt{\frac{k}{m} \cdot \frac{1+M / m}{M / m}} \cong \sqrt{\frac{k}{m}}$, and the corresponding eigenvectors are $\{z v\}=\left\{\begin{array}{ll}1 & 1\end{array}\right\}$ and $\{m / M-1\} \cong\{0-1\}$, respectively. ${ }^{[8]}$ Thus, the system moves as a rigid body that oscillates with the frequency $\omega_{2}$ when no external force exists (or after $t=1$ ). Here, it should be noted that the natural frequency $\left(\omega_{2}\right)$ approaches the frequency of a single DOF system with a fixed boundary as the large mass ratio increases. This implies that the large mass acts as a fixed boundary although it is also moving. However, if the large mass ratio becomes extremely large, for instance $M / m \geq 10^{12}$, the value of $\ddot{v}$ becomes so small during the interval $0 \leq t \leq 1$ that a computer cannot precisely express the small value due to the limitations of bits, that is, the computer expresses the small value of $\ddot{v}$ as zero due to a truncation error. Meanwhile, the value of $\ddot{z}$ approaches $a=1$ according to equation (6) and the computer expresses the value as 1.0 for the same reason. This numerical phenomenon can be observed in Table 2 .

In Table 3 , the relative motions $(\ddot{w}, \dot{w}$, and $w)$ of the LM model are compared with those of the AF model when $M / m=10^{5}$. Table 3 shows that the relative motions of the two models present nearly identical values when an appropriate ground mass is input.

\subsection{Influence of Erroneous Absolute Motions on Relative Motions in the LM Model}

Using the same test models in Figs. 5(b) and 5(c) subjected to the ground acceleration time history in Fig. 6, the same aspects were tested as in the previous section. The analytical solutions are given in the Appendix.
Table 3. Comparison of the Relative Motions of the LM Model $\left(M / m=10^{5}\right)$ with Those of the AF Model in Fig. 5, which are Subjected to the Ground Acceleration in Fig. 5(a)

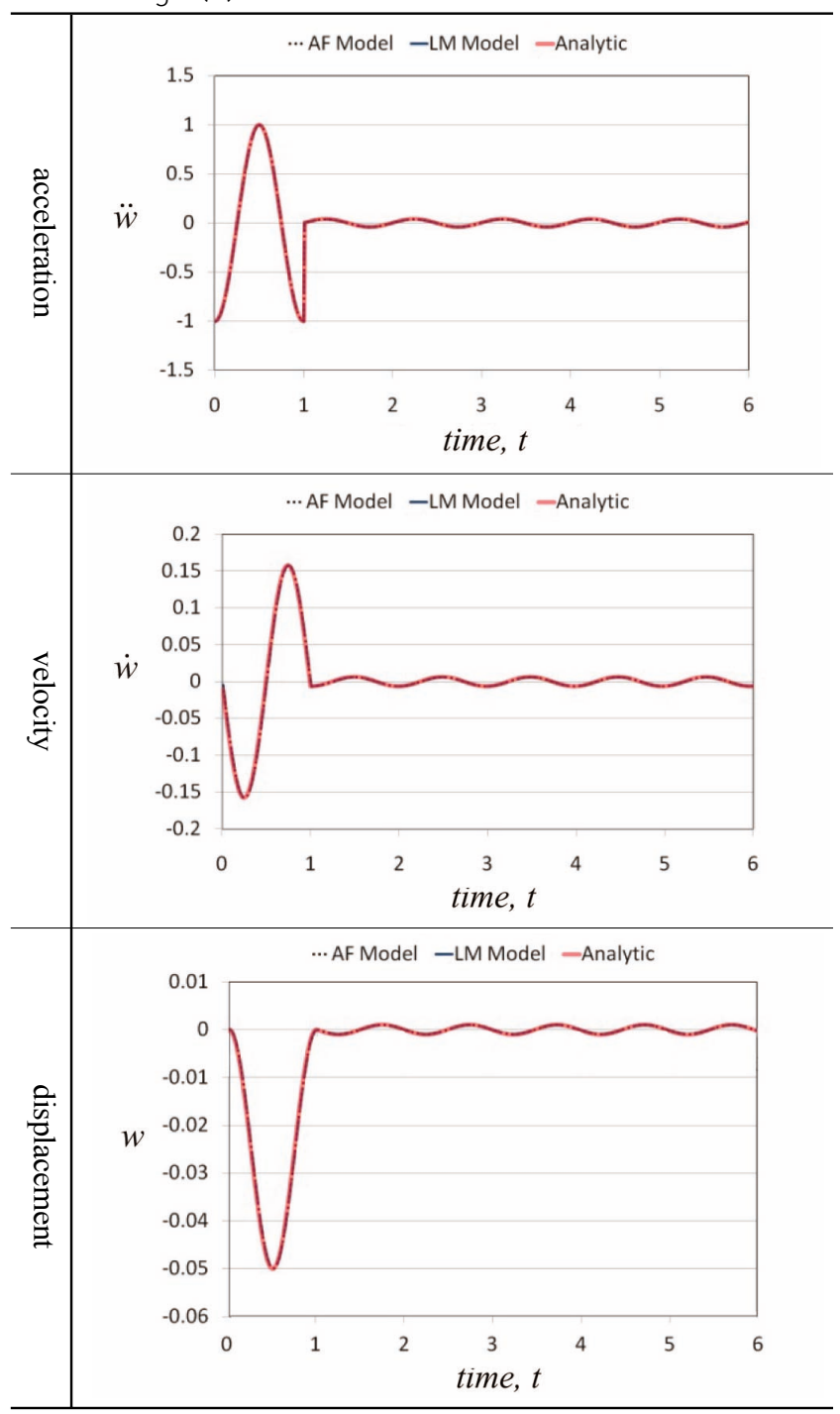

Table 2. Comparison of the Accelerations of the LM Model in Fig. 5(c) Subjected to the Ground Acceleration in Fig. 5(a) when M/m $=10^{11}$ and $10^{12}$

\begin{tabular}{|c|c|c|c|c|c|c|}
\hline \multirow[b]{2}{*}{$t$} & \multicolumn{2}{|c|}{$M / m=10^{11}$} & \multicolumn{2}{|c|}{$M / m=10^{12}$} & \multicolumn{2}{|c|}{ Analytic } \\
\hline & $\ddot{z}$ & $\ddot{v}$ & $\ddot{z}$ & $\ddot{v}$ & $\ddot{z}$ & $\ddot{v}$ \\
\hline 0.01 & 1.00000 & $0.100901 \mathrm{E}-2$ & 1.00000 & 0.00000 & 1.000000 & $0.199933 \mathrm{E}-2$ \\
\hline 0.2 & 1.00000 & 0.668855 & 1.00000 & 0.00000 & 1.000000 & 0.698862 \\
\hline 0.4 & 1.00000 & 1.79895 & 1.00000 & 0.00000 & 1.000000 & 1.81863 \\
\hline 0.6 & 1.00000 & 1.81274 & 1.00000 & 0.00000 & 1.000000 & 1.794179 \\
\hline 0.8 & 1.00000 & 0.691424 & 1.00000 & 0.00000 & 1.000000 & 0.659682 \\
\hline 1.0 & 1.00000 & $0.152310 \mathrm{E}-2$ & 1.00000 & 0.00000 & 1.000000 & $0.855604 \mathrm{E}-3$ \\
\hline
\end{tabular}


Table 4. Dynamic Responses of the LM Model in Fig. 5(c) Subjected to the Ground Acceleration in Fig. 6

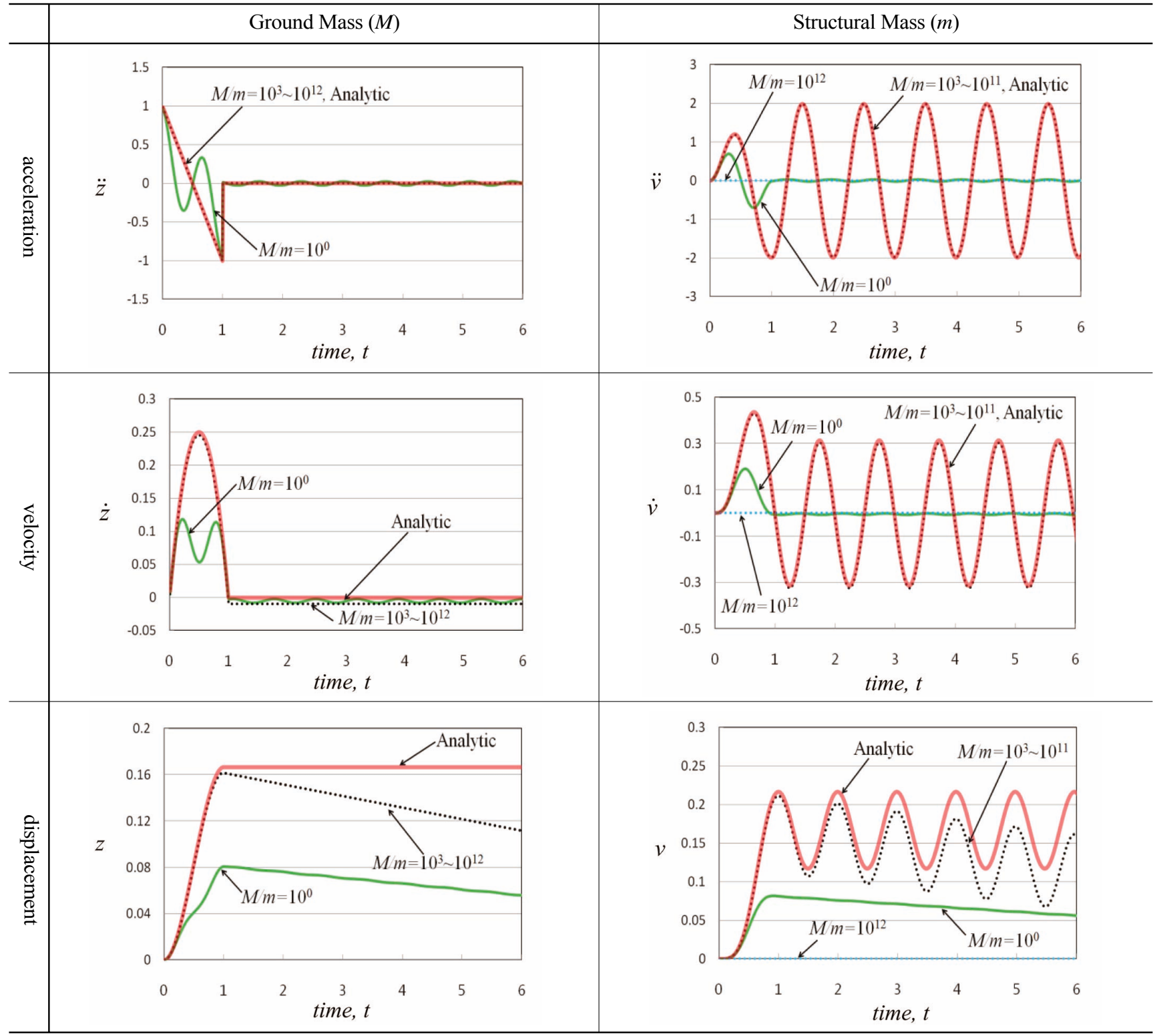

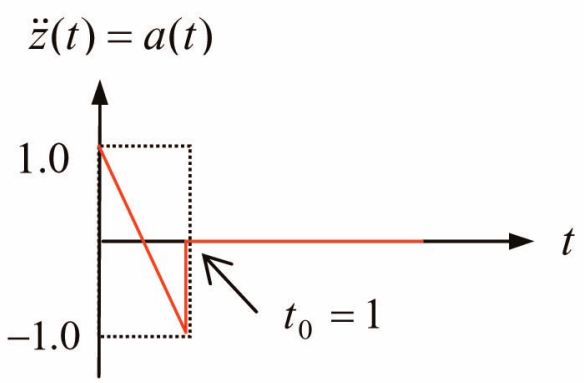

Fig. 6. Ground Acceleration Time History
Table 4 illustrates that the numerical behavior of the LM model depends on the mass ratio of $M / m$. As shown in Table $4, M / m=10^{\circ}$ yields erroneous responses, which results from the large mass ratio being too small to reproduce the given ground acceleration $a(t)$, as predicted in Theorem 1 and Theorem 4. The mass ratio in the range of $M / m=10^{3} \sim 10^{11}$ yields good approximate accelerations $(\ddot{z}$ and $\ddot{v}$ ) while the velocities $(\dot{z}$ and $\dot{v}$ ) deviate slightly from the analytical ones. Furthermore, the displacements ( $z$ and $v$ ) show significant errors, which will be discussed later in this section. The mass ratio of $M / m=10^{12}$ gives zero motion of the structural mass, that is, $\ddot{v}=0, \dot{v}=0$, 
and $v=0$. The accelerations $\ddot{z}$ and $\ddot{v}$ during the time interval $0 \leq t \leq 1$, in which $a(t) \neq 0$, are tabulated in Table 5 , and they are compared with the accelerations when $M / m=10^{11}$. The presence of an appropriate range of the large mass ratio was predicted by Theorem 3 . The physical meaning of the responses in the LM model, which depend on the large mass ratio, is identical to the explanations in the previous section.

In Table 6 , the relative motions ( $\ddot{w}, \dot{w}$, and $w$ ) of the AF model are compared with the relative motions calculated using the results of the LM model when $M / m=$ $10^{5}$. Table 6 shows that the LM model produces nearly identical relative motions to those of the AF model even when the absolute velocity $(\dot{z})$ and displacements $(z$ and $v)$ include errors. However, these errors can be reduced using a smaller time increment $(\Delta t)$. To demonstrate this, the same LM model was tested using different time increments when $\mathrm{M} / \mathrm{m}=10^{5}$. The results are plotted in Table 7, which shows that the errors originated from the size of the time increment of Newmark method of direct integration.
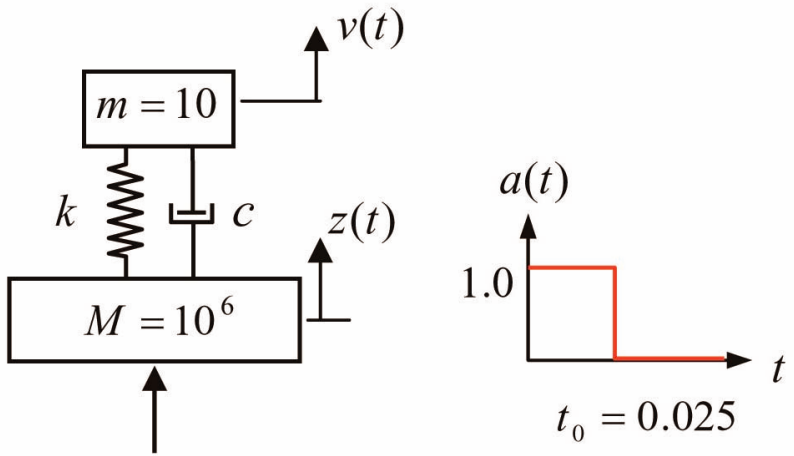

$F=M a(t)$

Fig. 7. The LM Model for Test where $M / m=10^{5}$
Table 6. Comparison of the Relative Motions of the LM Model $\left(M / m=10^{5}\right)$ with Those of the AF Model in Fig. 5, which are Subjected to the Ground Acceleration in Fig. 6

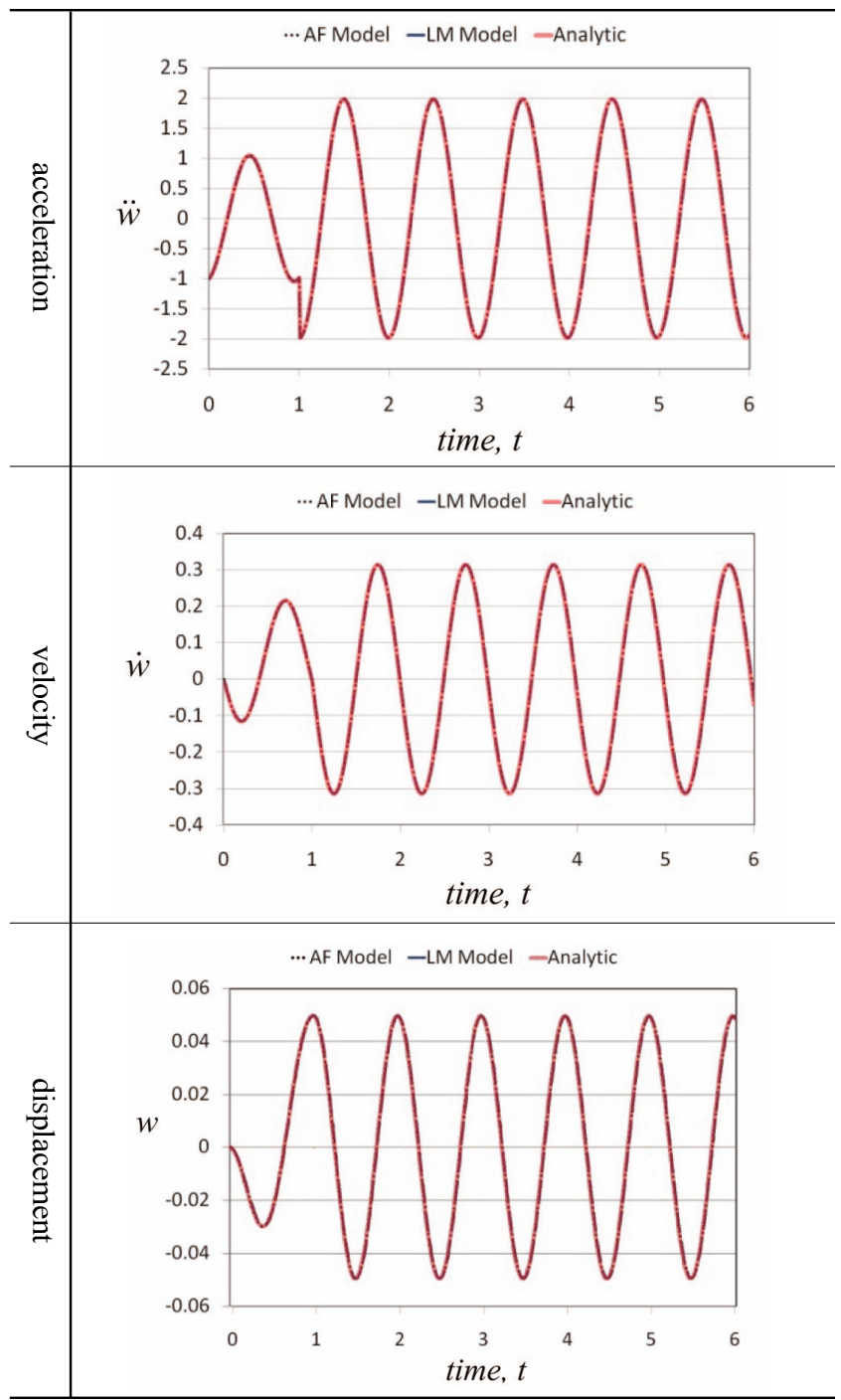

Table 5. Comparison of Accelerations of the LM Model in Fig. 5(c) Subjected to the Ground Acceleration in Fig. 6 when $\mathrm{M} / \mathrm{m}=10^{11}$ and $10^{12}$

\begin{tabular}{|c|c|c|c|c|c|c|}
\hline \multirow[b]{2}{*}{$t$} & \multicolumn{2}{|c|}{$M / m=10^{11}$} & \multicolumn{2}{|c|}{$M / m=10^{12}$} & \multicolumn{2}{|c|}{ Analytic } \\
\hline & $\ddot{z}$ & $\ddot{v}$ & $\ddot{z}$ & $\ddot{v}$ & $\ddot{z}$ & $\ddot{v}$ \\
\hline 0.01 & 0.980000 & $0.988826 \mathrm{E}-3$ & 0.980000 & 0.00000 & 0.980000 & $0.1986-\mathrm{E} 2$ \\
\hline 0.2 & 0.600000 & 0.570303 & 0.600000 & 0.00000 & 0.600000 & 0.600411 \\
\hline 0.4 & 0.200000 & 1.18071 & 0.200000 & 0.00000 & 0.200000 & 1.20025 \\
\hline 0.6 & -0.200000 & 0.421010 & -0.200000 & 0.00000 & -0.200000 & 0.402013 \\
\hline 0.8 & -0.600000 & -1.20587 & -0.600000 & 0.00000 & -0.600000 & -1.23767 \\
\hline 1.0 & -1.00000 & -1.98608 & -1.00000 & 0.00000 & -1.000000 & -1.98607 \\
\hline
\end{tabular}


Table 7. Dynamic Responses of the LM Model in Fig. 5(c) Subjected to the Ground Acceleration in Fig. 6, which are Dependent on the Time Interval $(\Delta t)$

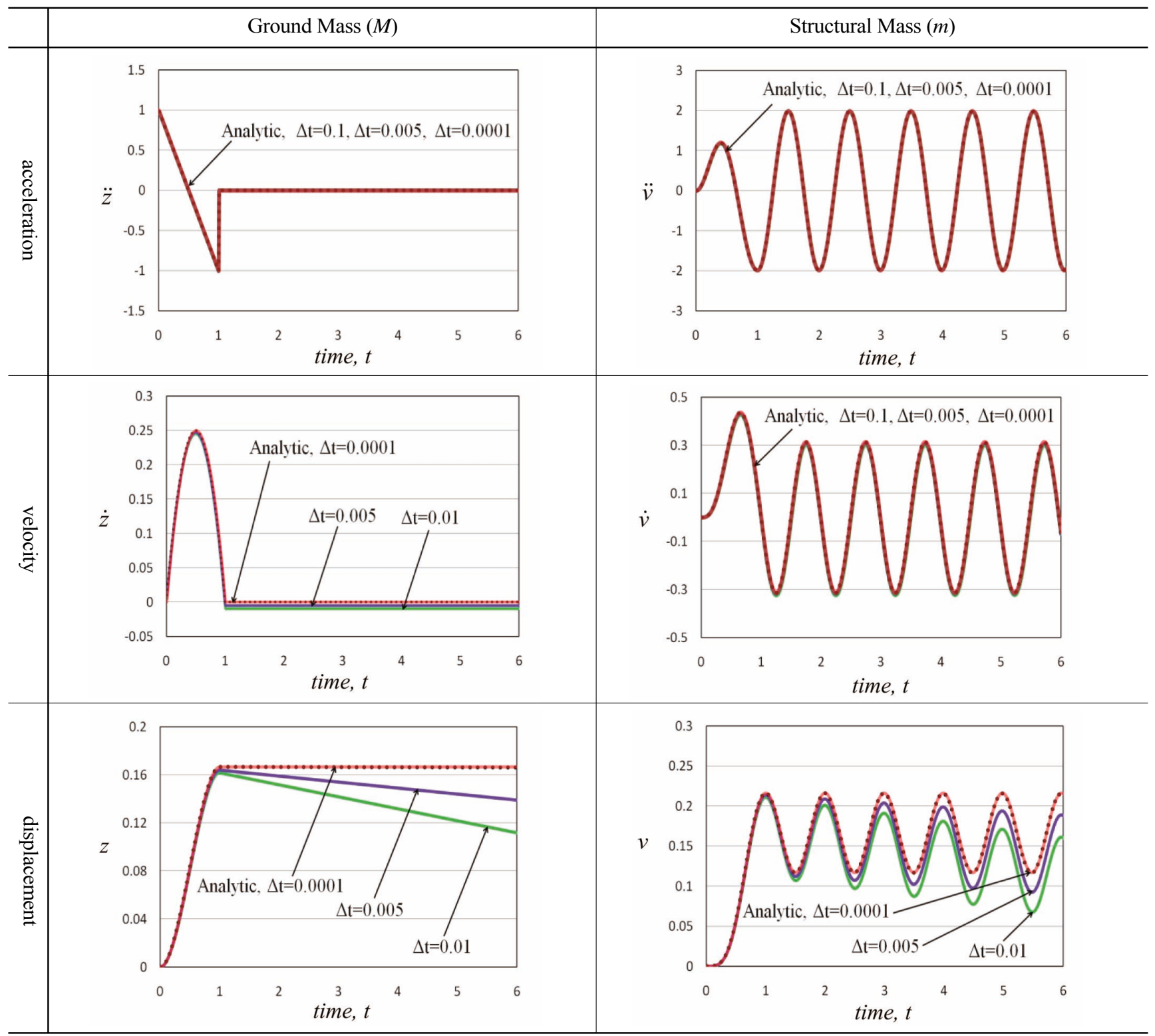

\subsection{Verification of Theorem 6}

To validate Theorem 6, numerical tests were performed by varying the magnitude of $k$ for the LM model in Fig. 7, where $m=10, M=10^{6}$, and $M / m=10^{5}$. The values of stiffness were $k=10^{4}, 10^{5}, 10^{6}, 10^{7}$, and $10^{8}$, as tabulated in Table 8 . The damping ratios of all cases in Table 8 are $\zeta=0.5$.

If Theorem 6 is incorrect, only Cases 1 and 2, which satisfy the conditions in equation (14), can produce good approximate solutions. Otherwise, all five cases will produce good approximate solutions.

The numerical results for the fifth case $\left(k / M=10^{2}\right)$ in
Table 8 are plotted in Table 9. The numerical responses in Table 9 agree with the analytical ones. The numerical results of the other cases in Table 8 also display accurate numerical behaviors. This indicates that the magnitudes of stiffness and the damping coefficient do not influence the accuracy of the numerical solutions if the large mass ratio is sufficiently large.

\subsection{Responses Compared with an Acceleration Time History of an Earthquake}

Thus far, simple acceleration time histories have been used to demonstrate the theorems. In this section, the real 
Table 8. Test Cases for Various Magnitudes of $k$ and Their Conditions in Eq. (14) for $M / m=10^{5}, m=10$, and $\zeta=0.5$

\begin{tabular}{c|c|c|c|c|c}
\hline Case & 1 & 2 & 3 & 4 & $10^{7}$ \\
\hline$k$ & $10^{4}$ & $10^{5}$ & $10^{6}$ & $10^{4}$ & $10^{8}$ \\
\hline$c=2 \zeta \sqrt{m k}$ & $10^{2.5}$ & $10^{3}$ & $10^{3.5}$ & $10^{1}$ & $10^{2}$ \\
\hline$k / M$ & $10^{-2}$ & $10^{-1}$ & $10^{0}$ & $10^{6}\left(>>\frac{M}{m}\right)$ & $10^{7}\left(>>\frac{M}{m}\right)$ \\
\hline$\omega_{n}{ }^{2}$ & $10^{3}\left(<<\frac{M}{m}\right)$ & $10^{4}\left(<<\frac{M}{m}\right)$ & $10^{5}\left(=\frac{M}{m}\right)$ & $10^{3}\left(<<\frac{M}{m}\right)$ & $10^{3.5}\left(<<\frac{M}{m}\right)$ \\
\hline $2 \zeta \omega_{n}$ & $10^{1.5}\left(<<\frac{M}{m}\right)$ & $10^{2}\left(<<\frac{M}{m}\right)$ & $10^{2.5}\left(<<\frac{M}{m}\right)$ & \\
\hline
\end{tabular}

Table 9. Dynamic Responses of the LM Model in Fig. 7 for $k / M=10^{2}$ in Table 8

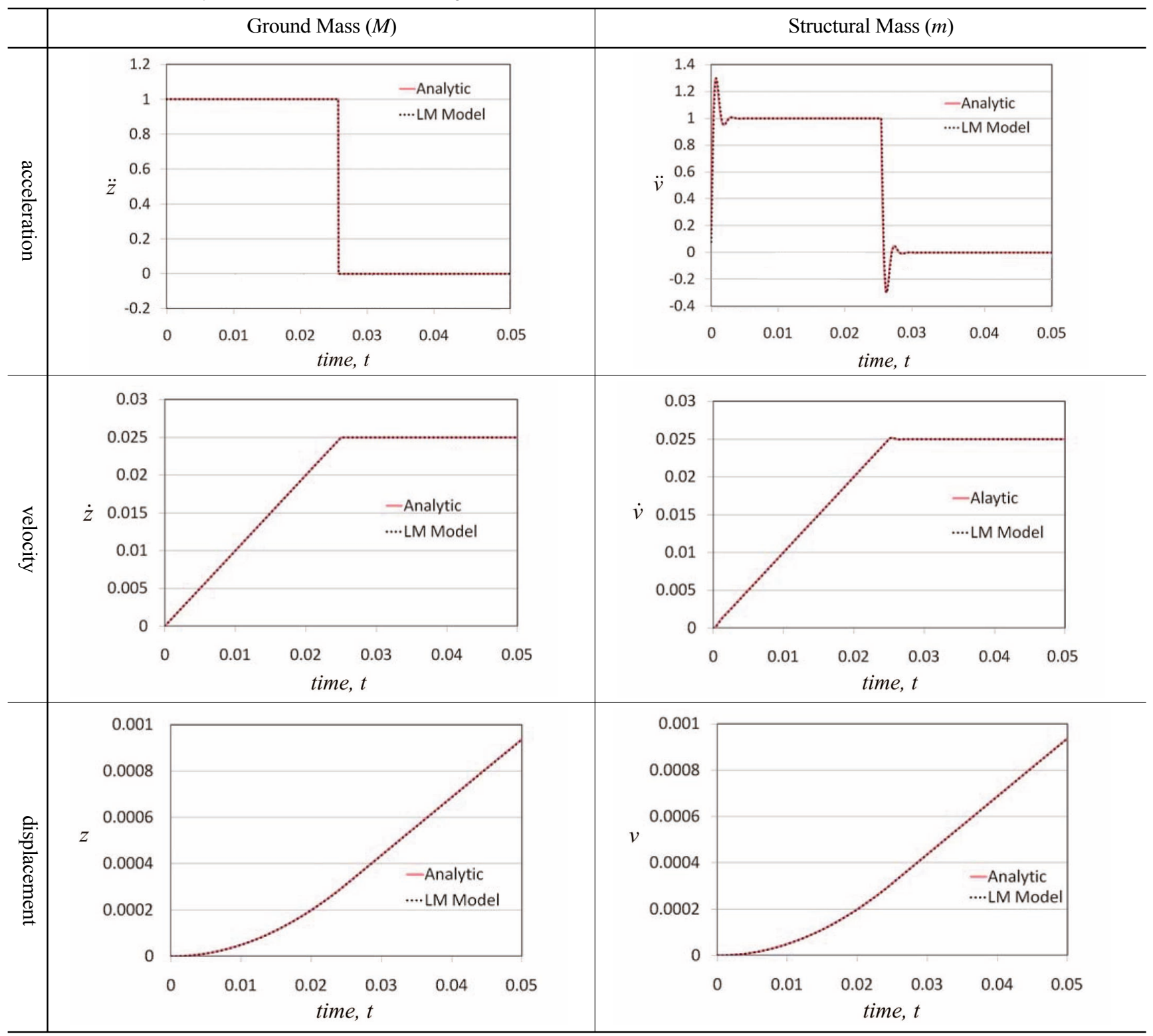


Table 10. Dynamic Responses of the LM Model in Fig. 5(c) Excited by the Acceleration Time History of the El Centro Earthquake when $\mathrm{M} / \mathrm{m}=10^{5}$

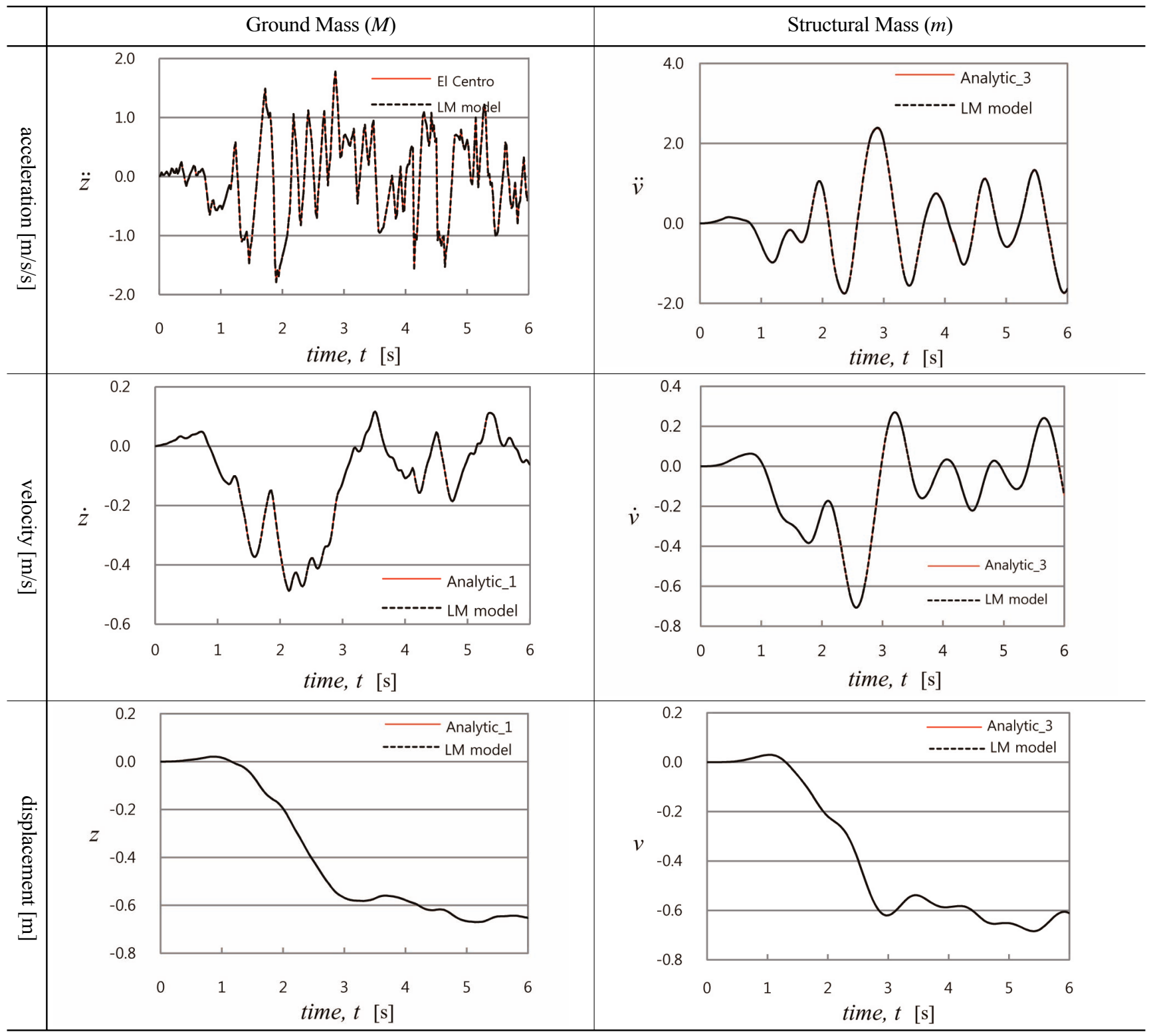

seismic acceleration time history of a ground, which is the east-west direction time history data from the El Centro site in the Imperial Valley irrigation district on May 18, 1940, is employed to demonstrate that the theorems hold well for real earthquake-induced ground accelerations. The acceleration time history during the first six seconds, which is shown in Fig. 8, is used to verify the accuracy of the solutions of the LM model and AF model.

Applying the time history data into the single DOF LM model and AF model in Fig. 5, the results were plotted as in Table 10 and Table 11 . Table 10 shows that the absolute motions of the ground and the structural mass $(\mathrm{m})$ agree with the analytic solutions. The analytic solutions designated by 'Analytic_1' were calculated using numerical integration of the acceleration time history of the El Centro earthquake in Fig. 8. Those for 'Analytic 3' were obtained by summing 'Analytic_1' and "Analytic_2", which appear in Table 11. The "Analytic_2" data are the relative motions $(\ddot{w}, \dot{w}, w)$ calculated using the convolution integral.

Table 11 shows three sets of relative motions: the 'LM model' set obtained by subtracting the ground 


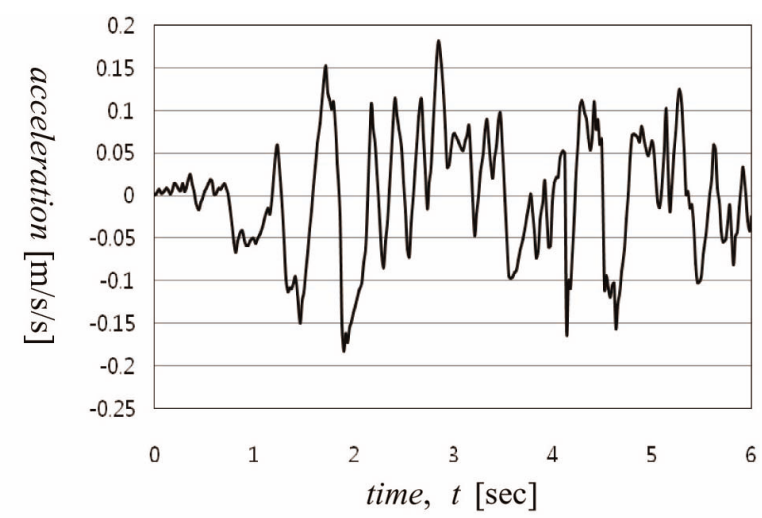

Fig. 8. The East-west Direction Accelerogram for the First Six Seconds Recorded at the El Centro Site in the Imperial Valley Irrigation District on May 18, 1940.

Table 11. Comparison of the Relative Motions of the LM Model $\left(\mathrm{M} / \mathrm{m}=10^{5}\right)$ with Those of the AF Model in Fig. 5, which are Subjected to the Ground Acceleration Time History of the El Centro Earthquake.

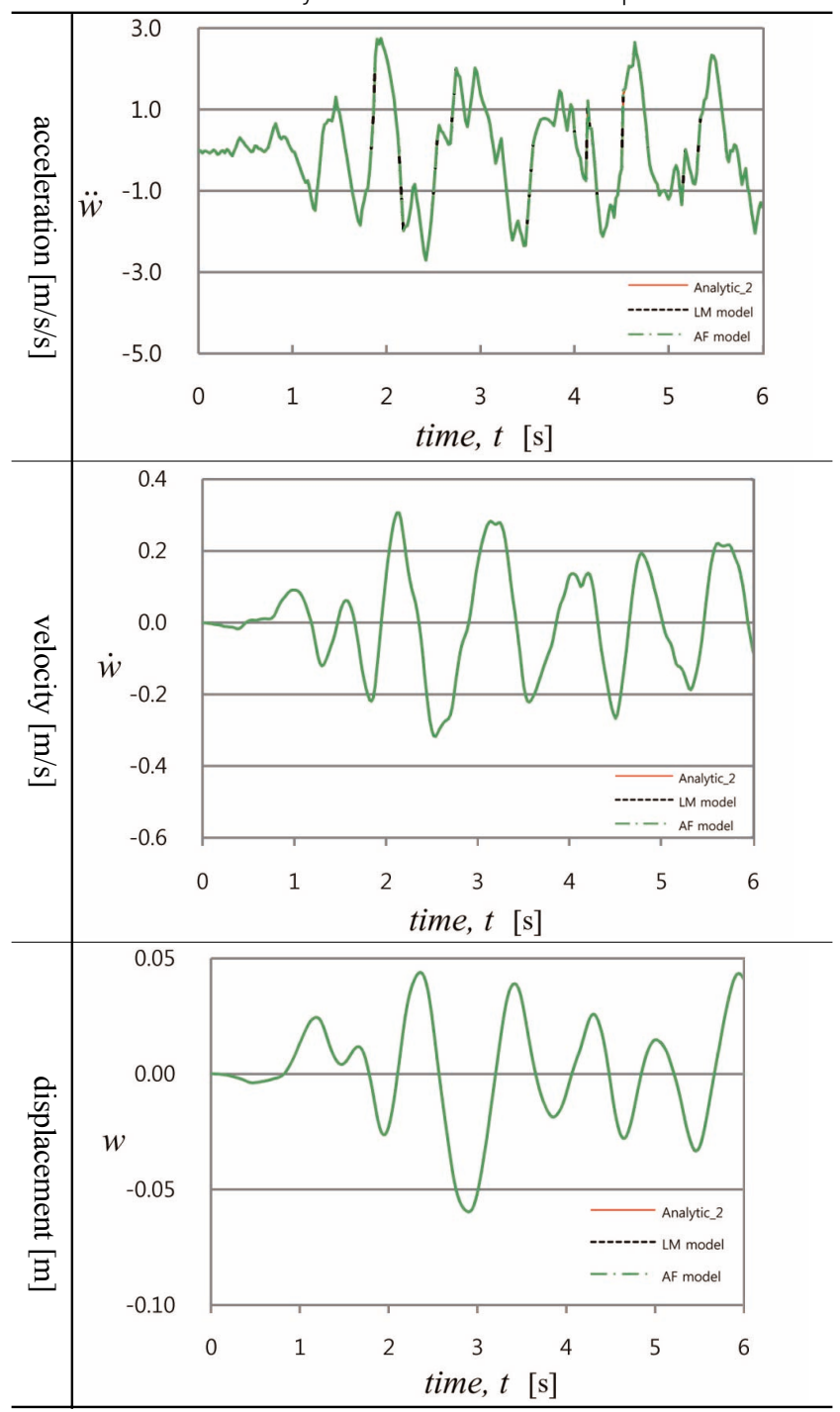

motions from the absolute motions of the structural mass, the 'AF model' set obtained using the AF model, and the 'Analytic 2' set calculated using the convolution integral. The three sets of relative motions agree well with each another. Thus, all motions in Table 10 and Table 11 are accurate motions of the ground and structural mass, even when the El Centro acceleration time history is employed for the excitation.

\section{CONCLUSION}

Useful theorems on the large mass (LM) model are presented through a mathematical analysis of the equations of motion of a structure subjected to a ground acceleration time history. The analysis provides insight into how the LM model yields good or erroneous solutions depending on the ratio of the ground mass to the structural mass. It also shows that the solution's erroneous behavior in the LM model is not related to the magnitudes of stiffness and the damping coefficient. Numerical tests confirmed the theorems and demonstrated that the large mass ratio should not be too small or too large in order to produce good approximate solutions. The velocity and displacement responses of the LM model also showed numerical errors if the time increment of the direct integration was not sufficiently small. However, the relative motions obtained using the motions of the LM model are accurate if the large mass ratio is appropriately large.

In contrast to the LM model, the AF model produced accurate relative motions and does not require an artificial parameter to be input. Thus, the AF model is recommended over than LM model when only the relative motions are sought for the system excited by a ground acceleration time history.

\section{APPENDIX}

\section{(i) Analytic Solutions for the System Subjected to the Acceleration in Fig. 5:}

Assume that initial conditions are given by:

$$
z(0)=0, v(0)=0, \dot{z}(0)=0 \text {, and } \dot{v}(0)=0,
$$

and the ground acceleration history is given as:

$$
\ddot{z}(t)= \begin{cases}1 & \text { for } 0 \leq t \leq t_{0} \\ 0 & \text { for } t>t_{0}\end{cases}
$$

By successive integration of $\ddot{z}(t)$ in equation (A2), the following velocity and displacement of the ground are obtained:

$$
\dot{z}(t)= \begin{cases}t & \text { for } 0 \leq t \leq t_{0} \\ t_{0} & \text { for } t>t_{0}\end{cases}
$$




$$
z(t)= \begin{cases}0.5 t^{2} & \text { for } 0 \leq t \leq t_{0} \\ t_{0} t-0.5 t_{0}^{2} & \text { for } t>t_{0}\end{cases}
$$

The equation of motion of the system in Fig. 5 is expressed as:

$$
m \ddot{w}+k w=-m \ddot{z},
$$

where

$$
w=v-z .
$$

Using equations (A1) and (A6), the initial conditions of equation (A5) are obtained as follows:

$$
w(0)=0, \dot{w}(0)=0 .
$$

Applying a convolution integral ${ }^{[9]}$ to equation (A5), the following solutions are obtained:

$$
\begin{gathered}
w(t)=\left\{\begin{array}{lr}
\frac{m}{k}\left\{\cos \left(\omega_{n} t\right)-1\right\} & \text { for } \quad 0 \leq t \leq t_{0}, \\
\frac{m}{k}\left[\cos \left(\omega_{n} t\right)-\cos \left\{\omega_{n}\left(t-t_{0}\right)\right\}\right. & \text { for } t>t_{0}
\end{array},\right. \\
\dot{w}(t)= \begin{cases}-\left(\frac{m}{k}\right)^{0.5} \sin \left(\omega_{n} t\right) & \text { for } 0 \leq t \leq t_{0} \\
-\left(\frac{m}{k}\right)^{0.5}\left[\sin \left(\omega_{n} t\right)-\sin \left\{\omega_{n}\left(t-t_{0}\right)\right\}\right] & \text { for } t>t_{0}\end{cases} \\
\ddot{w}(t)= \begin{cases}-\cos \left(\omega_{n} t\right) & \text { for } \quad 0 \leq t \leq t_{0}, \\
-\left[\cos \left(\omega_{n} t\right)-\cos \left\{\omega_{n}\left(t-t_{0}\right)\right\}\right] & \text { for } t>t_{0}\end{cases}
\end{gathered}
$$

where $\omega_{n}=\sqrt{k / m}$.

Then, using the relation $v=z+w$, the following motions of structural mass are obtained:

$$
\begin{aligned}
& v(t)= \begin{cases}\frac{1}{2} t^{2}+\frac{m}{k}\left\{\cos \left(\omega_{n} t\right)-1\right\} & \text { for } \quad 0 \leq t \leq t_{0} \\
t_{0} t-\frac{1}{2} t_{0}{ }^{2}+\frac{m}{k}\left[\cos \left(\omega_{n} t\right)\right. & \text { for } t>t_{0}\end{cases} \\
& \dot{v}(t)= \begin{cases}t-\left(\frac{m}{k}\right)^{0.5} \sin \left(\omega_{n} t\right) & \text { for } \quad 0 \leq t \leq t_{0} \\
t_{0}-\left(\frac{m}{k}\right)^{0.5}\left[\sin \left(\omega_{n} t\right)-\sin \left\{\omega_{n}\left(t-t_{0}\right)\right\}\right] & \text { for } t>t_{0}\end{cases} \\
& \ddot{v}(t)=\left\{\begin{array}{lr}
1-\cos \left(\omega_{n} t\right) & \text { for } \quad 0 \leq t \leq t_{0} \\
-\left[\cos \left(\omega_{n} t\right)-\cos \left\{\omega_{n}\left(t-t_{0}\right)\right\}\right] & \text { for } t>t_{0}
\end{array}\right.
\end{aligned}
$$

\section{(ii) Analytic Solutions for the System Subjected to the Acceleration in Fig. 6:}

For the same initial conditions as equation (A1), the solution of equation (A5) subjected to following ground acceleration history is obtained.

$$
\ddot{z}(t)=\left\{\begin{array}{lll}
-\frac{2}{t_{0}} t+1 & \text { for } & 0 \leq t \leq t_{0} \\
0 & \text { for } t>t_{0}
\end{array} .\right.
$$

Integrating the above equation (A14) successively, the following is obtained:

$$
\begin{aligned}
& \dot{z}(t)=\left\{\begin{array}{lll}
-\frac{1}{t_{0}} t^{2}+t & \text { for } & 0 \leq t \leq t_{0} \\
0 & \text { for } & t>t_{0}
\end{array},\right.
\end{aligned}
$$

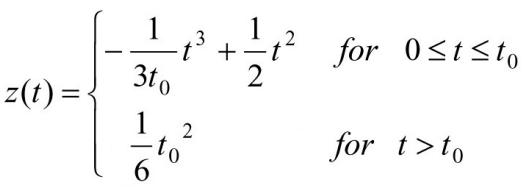

Applying the convolution integral to the equation of motion (A5) with the initial conditions in equation (A7), the following solutions are obtained:

$$
\begin{aligned}
& \left(\frac{m}{k}\right)\left(\cos \left(\omega_{n} t\right)+\frac{2}{t_{0}} t-1\right) \\
& w(t)=\left\{-\left(\frac{m}{k}\right)^{1.5}\left(\frac{2}{t_{0}}\right) \sin \left(\omega_{n} t\right)\right. \\
& -\frac{2}{t_{0}}\left(\frac{m}{k}\right)^{1.5}\left[\sin \left(\omega_{n} t\right)-\sin \left\{\omega_{n}\left(t-t_{0}\right)\right\}\right] \\
& +\left(\frac{m}{k}\right)\left[\cos \left(\omega_{n} t\right)+\cos \left\{\omega_{n}\left(t-t_{0}\right)\right\}\right] \\
& \text { for } 0 \leq t \leq t_{0} \\
& \text { for } t>t_{0} \\
& -\left(\frac{m}{k}\right)^{0.5} \sin \left(\omega_{n} t\right) \\
& \dot{w}(t)=\left\{\begin{array}{ll}
+\left(\frac{m}{k}\right)\left(\frac{2}{t_{0}}\right)\left\{1-\cos \left(\omega_{n} t\right)\right\} & \text { for } 0 \leq t \leq t_{0} \\
-\frac{2}{t_{0}}\left(\frac{m}{k}\right)\left[\cos \left(\omega_{n} t\right)-\cos \left\{\omega_{n}\left(t-t_{0}\right)\right\}\right] & \text { for } t>t_{0} \\
-\left(\frac{m}{k}\right)^{0.5}\left[\sin \left(\omega_{n} t\right)+\sin \left\{\omega_{n}\left(t-t_{0}\right)\right\}\right] &
\end{array},\right. \\
& \dot{w}(t)=\left\{\begin{array}{ll}
+\left(\frac{m}{k}\right)\left(\frac{2}{t_{0}}\right)\left\{1-\cos \left(\omega_{n} t\right)\right\} & \text { for } 0 \leq t \leq t_{0} \\
-\frac{2}{t_{0}}\left(\frac{m}{k}\right)\left[\cos \left(\omega_{n} t\right)-\cos \left\{\omega_{n}\left(t-t_{0}\right)\right\}\right] & \text { for } t>t_{0} \\
-\left(\frac{m}{k}\right)^{0.5}\left[\sin \left(\omega_{n} t\right)+\sin \left\{\omega_{n}\left(t-t_{0}\right)\right\}\right] &
\end{array},\right. \\
& \dot{w}(t)=\left\{\begin{array}{ll}
+\left(\frac{m}{k}\right)\left(\frac{2}{t_{0}}\right)\left\{1-\cos \left(\omega_{n} t\right)\right\} & \text { for } 0 \leq t \leq t_{0} \\
-\frac{2}{t_{0}}\left(\frac{m}{k}\right)\left[\cos \left(\omega_{n} t\right)-\cos \left\{\omega_{n}\left(t-t_{0}\right)\right\}\right] & \text { for } t>t_{0} \\
-\left(\frac{m}{k}\right)^{0.5}\left[\sin \left(\omega_{n} t\right)+\sin \left\{\omega_{n}\left(t-t_{0}\right)\right\}\right] &
\end{array},\right. \\
& \dot{w}(t)=\left\{\begin{array}{ll}
+\left(\frac{m}{k}\right)\left(\frac{2}{t_{0}}\right)\left\{1-\cos \left(\omega_{n} t\right)\right\} & \text { for } 0 \leq t \leq t_{0} \\
-\frac{2}{t_{0}}\left(\frac{m}{k}\right)\left[\cos \left(\omega_{n} t\right)-\cos \left\{\omega_{n}\left(t-t_{0}\right)\right\}\right] & \text { for } t>t_{0} \\
-\left(\frac{m}{k}\right)^{0.5}\left[\sin \left(\omega_{n} t\right)+\sin \left\{\omega_{n}\left(t-t_{0}\right)\right\}\right] &
\end{array},\right. \\
& \dot{w}(t)=\left\{\begin{array}{ll}
+\left(\frac{m}{k}\right)\left(\frac{2}{t_{0}}\right)\left\{1-\cos \left(\omega_{n} t\right)\right\} & \text { for } 0 \leq t \leq t_{0} \\
-\frac{2}{t_{0}}\left(\frac{m}{k}\right)\left[\cos \left(\omega_{n} t\right)-\cos \left\{\omega_{n}\left(t-t_{0}\right)\right\}\right] & \text { for } t>t_{0} \\
-\left(\frac{m}{k}\right)^{0.5}\left[\sin \left(\omega_{n} t\right)+\sin \left\{\omega_{n}\left(t-t_{0}\right)\right\}\right] &
\end{array},\right. \\
& \dot{v}(t)=\left\{\begin{array}{ll}
-\cos \left(\omega_{n} t\right)+\left(\frac{m}{k}\right)^{0.5}\left(\frac{2}{t_{0}}\right) \sin \left(\omega_{n} t\right) \quad \text { for } & 0 \leq t \leq t_{0} \\
\frac{2}{t_{0}}\left(\frac{m}{k}\right)^{0.5}\left[\sin \left(\omega_{n} t\right)-\sin \left\{\omega_{n}\left(t-t_{0}\right)\right\}\right] & \text { for } t>t_{0} \\
-\left[\cos \left(\omega_{n} t\right)+\cos \left\{\omega_{n}\left(t-t_{0}\right)\right\}\right] &
\end{array} .\right.
\end{aligned}
$$

Then, using the relation $v=z+w$, the motion of structural mass is obtained as follows: 


$$
\begin{aligned}
& \left(-\frac{1}{3 t_{0}} t^{3}+\frac{1}{2} t^{2}+\left(\frac{m}{k}\right)\left(\cos \left(\omega_{n} t\right)+\frac{2}{t_{0}} t-1\right)\right. \\
& -\left(\frac{m}{k}\right)^{1.5}\left(\frac{2}{t_{0}}\right) \sin \left(\omega_{n} t\right) \quad \text { for } 0 \leq t \leq t_{0} \\
& \frac{t_{0}{ }^{2}}{6}-\frac{2}{t_{0}}\left(\frac{m}{k}\right)^{1.5}\left[\sin \left(\omega_{n} t\right)-\sin \left\{\omega_{n}\left(t-t_{0}\right)\right\}\right] \\
& +\left(\frac{m}{k}\right)\left[\cos \left(\omega_{n} t\right)+\cos \left\{\omega_{n}\left(t-t_{0}\right)\right\}\right] \\
& -\frac{1}{t_{0}} t^{2}+t-\left(\frac{m}{k}\right)^{0.5} \sin \left(\omega_{n} t\right) \quad \text { for } 0 \leq t \leq t_{0} \\
& \dot{v}(t)=\left\{+\left(\frac{m}{k}\right)\left(\frac{2}{t_{0}}\right)\left\{1-\cos \left(\omega_{n} t\right)\right\},\right. \\
& -\frac{2}{t_{0}}\left(\frac{m}{k}\right)\left[\cos \left(\omega_{n} t\right)-\cos \left\{\omega_{n}\left(t-t_{0}\right)\right\}\right] \quad \text { for } t>t_{0} \\
& -\left(\frac{m}{k}\right)^{0.5}\left[\sin \left(\omega_{n} t\right)+\sin \left\{\omega_{n}\left(t-t_{0}\right)\right\}\right] \\
& \ddot{v}(t)=\left\{\begin{array}{lr}
-\frac{2}{t_{0}} t+1-\cos \left(\omega_{n} t\right)+\left(\frac{m}{k}\right)^{0.5}\left(\frac{2}{t_{0}}\right) \sin \left(\omega_{n} t\right) & \text { for } 0 \leq t \leq t_{0} \\
-\left[\cos \left(\omega_{n} t\right)+\cos \left\{\omega_{n}\left(t-t_{0}\right)\right\}\right] & \text { (A22) } \\
+\frac{2}{t_{0}}\left(\frac{m}{k}\right)^{0.5}\left[\sin \left(\omega_{n} t\right)-\sin \left\{\omega_{n}\left(t-t_{0}\right)\right\}\right] & \text { for } t>t_{0}
\end{array} .\right.
\end{aligned}
$$

[ 8 ] R. D. Blevins, Formulas for Natural Frequency and Mode Shape, Van Nostrand Reinhold, New York, 1979

[9] S. S. Rao, Mechanical Vibrations, Second Edition, Addison Wesley, 1990 